\title{
Prognostic utility of HOXBI 3: ILI7BR and molecular grade index in early-stage breast cancer patients from the Stockholm trial
}

\section{P-L Jerevall',I, X-J Ma ${ }^{2}$, L Li$^{2}$, R Salunga ${ }^{2}$, NC Kesty ${ }^{2}$, MG Erlander ${ }^{2}$, DC Sgroi ${ }^{3}$, B Holmlund', L Skoog ${ }^{4}$, T Fornander ${ }^{5}$, B Nordenskjöld' ' and O Stål'}

'Department of Clinical and Experimental Medicine, Division of Oncology, Faculty of Health Sciences, Linköping University, Linköping, SE58 I 85, Sweden; ${ }^{2}$ bioTheranostics, 9640 Towne Centre Dr Suite 200, San Diego, CA 92121 , USA; ${ }^{3}$ Department of Pathology, Molecular Pathology Research Unit, Massachusetts General Hospital, Boston, MA 021 29, USA; ${ }^{4}$ Department of Oncology, Karolinska University Hospital, Stockholm South General Hospital, Stockholm, SEI I 883, Sweden; ${ }^{5}$ Department of Pathology and Cytology, Karolinska University Hospital, Solna, Stockholm SE I 7 I 76, Sweden

BACKGROUND: A dichotomous index combining two gene expression assays, HOXBI3:ILI7BR (H:I) and molecular grade index (MGl), was developed to assess risk of recurrence in breast cancer patients. The study objective was to demonstrate the prognostic utility of the combined index in early-stage breast cancer.

METHODS: In a blinded retrospective analysis of 588 ER-positive tamoxifen-treated and untreated breast cancer patients from the randomised prospective Stockholm trial, $\mathrm{H}: \mathrm{I}$ and MGl were measured using real-time RT-PCR. Association with patient outcome was evaluated by Kaplan-Meier analysis and Cox proportional hazard regression. A continuous risk index was developed using Cox modelling.

RESULTS: The dichotomous $\mathrm{H}: \mathrm{I}+\mathrm{MGI}$ was significantly associated with distant recurrence and breast cancer death. The $>50 \%$ of tamoxifen-treated patients categorised as low-risk had $<3 \%$ I0-year distant recurrence risk. A continuous risk model (Breast Cancer Index $(\mathrm{BCl})$ ) was developed with the tamoxifen-treated group and the prognostic performance tested in the untreated group was $53 \%$ of patients categorised as low risk with an $8.3 \%$ 10-year distant recurrence risk.

CONCLUSION: Retrospective analysis of this randomised, prospective trial cohort validated the prognostic utility of $\mathrm{H}: I+\mathrm{MGl}$ and was used to develop and test a continuous risk model that enables prediction of distant recurrence risk at the patient level.

British Journal of Cancer (201 I) I 04, 1762-1769. doi:10.1038/bjc.2011.145 www.bjcancer.com

Published online 10 May 201 I

(c) 201। Cancer Research UK

Keywords: Breast Cancer Index; recurrence; risk assessment; gene expression profiling; prognosis

Conventional criteria used for risk prediction of breast cancer patients include clinicopathological characteristics such as lymph node involvement, tumour size, histological tumour grade and hormone receptor status (Carter et al, 1989; Galea et al, 1992; Press et al, 1997). However, even with utilisation of these prognostic and treatment predictive factors under and overtreatment can occur (van't Veer and Bernards, 2008). Using traditional risk classifications, a large proportion of patients are classified as intermediate risk, which is uninformative for choosing optimal treatment strategies for the individual patients. Currently, the selection criteria for using or withholding chemotherapy are coarsely defined; however, this selection is of major importance in order to avoid unnecessary toxic side effects associated with therapy (Goldhirsch et al, 2009). Adjuvant treatment decisions based on clinicopathological characteristics as recommended by guidelines, such as the St Gallen breast cancer consensus guidelines and the Adjuvant! Online tool (http://www.adjuvantonline.com), can be used for assessing risks and benefits associated with adjuvant therapy (Ravdin et al, 2001; Goldhirsch et al, 2006, 2009).

*Correspondence: Dr P-L Jerevall; E-mail: piiha-lotta.jerevall@liu.se Received 2 February 2011; revised 31 March 2011; accepted 4 April 20I I; published online I0 May 20II
With the development of prognostic and predictive gene expression signatures, clinicians can be provided with information beyond the traditional criteria to guide treatment selection. Adjunctive to other standard risk factors, such as tumour size, tumour grade, hormone receptor status and nodal status, integration of gene expression signatures in the clinic provides the potential to more accurately identify low- and high-risk patients for better informed treatment-decision making. Despite a lack of overlapping genes in different predictive gene signatures, the risk classification of different tumours can be equivalent between different signatures (Desmedt and Sotiriou, 2006; Fan et al, 2006), whereas it is evident that using conventional criteria, as compared with these novel risk stratification tools, a significant proportion of the patients are misclassified (van de Vijver et al, 2002; Goldstein et al, 2008).

In a previous study, it was demonstrated that the combination of HOXB13:IL17BR (H:I) and molecular grade index (MGI), two independent prognostic markers, outperformed either index alone in predicting risk of recurrence in breast cancer patients (Ma et al, 2004, 2008). HOXB13: IL17BR is an independent prognostic factor for patients with oestrogen receptor (ER) positive and node negative disease that has also been shown to be a negative predictive factor of tamoxifen benefit (Ma et al, 2004, 2006; Goetz et al, 2006; Jerevall et al, 2008). HOXB13 separately can also 
identify patients with limited benefit of endocrine treatment. This has been shown for gene expression, as well as protein levels (Jerevall et al, 2008, 2010). Molecular grade index is a gene expression assay, comprised of five genes related to histological grade and tumour progression, which recapitulates tumour grade and can predict clinical outcome with high performance (Ma et al, 2008). With the combinatorial approach of $\mathrm{H}$ : I and MGI, it was shown that breast cancer patients could be stratified into three risk groups with better risk prediction of distant metastasis in ER positive, lymph node-negative patients.

In this study, we have further validated the prognostic utility of the dichotomous $\mathrm{H}: \mathrm{I}$ and MGI index retrospectively in a large patient cohort from the prospective randomized Stockholm trial of low risk, ER positive, node negative, tamoxifen-treated or untreated patients (Rutqvist and Johansson, 2007). In addition, to facilitate individualised risk assessment in the clinical setting, a continuous predictor based on the $\mathrm{H}: \mathrm{I}$ and MGI referred to as the Breast Cancer Index (BCI) was developed and tested. Within this cohort from the Stockholm trial, BCI is shown to predict risk of recurrence at the individual level.

\section{PATIENTS AND METHODS}

\section{Patients and tumour samples}

The Stockholm breast cancer study group conducted a randomised tamoxifen trial during 1976 through 1990 within a total of 2738 postmenopausal women with invasive early-stage disease (Rutqvist and Johansson, 2007). The trial included a lymph node negative low-risk group comprising 1780 patients with tumours $\leqslant 30 \mathrm{~mm}$ in diameter, randomised to 2 years of adjuvant tamoxifen $(40 \mathrm{mg}$ daily) vs control. The control patients were systemically untreated and did not receive any chemotherapy. In 1983, a new trial was initiated, in which recurrence-free patients, after 2 years of tamoxifen treatment, were randomised to 3 more years of tamoxifen or no further therapy. As a result of the new trial, the patients in the tamoxifen arm were treated either for 2 or 5 years. In the Stockholm cohort, the benefit from tamoxifen was largely independent of treatment duration (Rutqvist and Johansson, 2007).

For this study, tumour blocks from 808 patients were received (tamoxifen treated (2-5 years) and untreated). As tumour grade was not determined during the actual trial, it was determined retrospectively, by one pathologist blinded to outcome. The tumours were graded according to the Nottingham system. After pathology review, 37 cases were excluded because of insufficient number of tumour cells in the sample, or only containing carcinoma in situ. The remaining subset (771 tumour blocks) was well balanced to the original low-risk cohort regarding the tumour characteristics, such as tumour size of $\leqslant 20 \mathrm{~mm}$ (78 vs $81 \%$ ), positive ER status (78 vs $80 \%$ ) and tamoxifen treatment (52 vs 50\%).

The standard procedure for tissue collection was fixation in $4 \%$ phosphate-buffered formalin and embedment in paraffin. Followup data were collected from regional population registers and the Swedish Cause of Death Registry. The mean follow-up period for patients in the present investigation was 17 years. The retrospective investigation of the collected tumour samples was approved by the ethical committee at the Karolinska University Hospital. According to the approval, informed consent from the patients was not required.

\section{Hormone receptor status}

Status of ER, progesterone receptor (PR) and human epidermal growth factor receptor 2 (HER2) was assessed retrospectively with immunohistochemistry. ER and PR were examined using the
Ventana automated slide stainer (Ventana Medical Systems, SA, Cedex, France). Primary monoclonal antibodies were mouse antiER antibody (clone 6F11) and mouse anti-PR antibody (clone 16). Cut-off level was set to $25 \%$ positively stained tumour cell nuclei. In cases when immunohistochemical data for ER were missing (12\%), ER status as determined in clinical routine practice at time of diagnosis was used (Wrange et al, 1978), with a cut-off level of $0.05 \mathrm{fmol} \mu \mathrm{g}^{-1}$ DNA. For HER2 status, tissue was stained and scored as previously described (Jerevall et al, 2010).

\section{Gene expression analysis by real-time RT-PCR and calculation of gene expression indices}

Molecular grade index, $\mathrm{H}: \mathrm{I}$ and $\mathrm{BCI}$ analysis were carried out blinded to outcome. The genes analysed were HOXB13, IL17BR (HOXB13:IL17BR index or $\mathrm{H}: \mathrm{I}$ ), BUB1B, CENPA, NEK2, RACGAP1, RRM2 (molecular grade index), ACTB, HMBS, SDHA and $U B C$ (reference genes). Primer and probe sequences for these genes were the same as previously described (Ma et al, $2006,2008)$. From each sample, $10 \mu \mathrm{m}$ tissue sections were cut. To enrich for tumour content, all sections were subjected to manual macrodissection before RNA extraction. RNA extraction from formalin-fixed paraffin-embedded (FFPE) sections was carried out as before (Ma et al, 2006). Prior to TaqMan RTPCR, total RNA was reverse transcribed, and the resulting cDNA was pre-amplified by performing 10 rounds of PCR using the PreAmp Master Mix Kit per manufacturer's instructions (Applied Biosystems, Carlsbad, CA, USA). The pre-amplified products were analysed by TaqMan RT-PCR as previously described (Ma et al, 2008). H: I and MGI were calculated as previously described (Ma et al, 2006, 2008).

\section{Development of a continuous risk model}

Previously, we reported the categorical combination of binary $\mathrm{H}$ : I (cut-off $=0.06$ ) and MGI (cut-off $=0$ ) into three risk groups as follows: low risk, low MGI; intermediate risk, low H: I and high MGI; and high risk, high $\mathrm{H}: \mathrm{I}$ and high MGI. Here a continuous risk model was built by combining $\mathrm{H}$ : I and MGI as continuous variables, using the ER-positive patients in the tamoxifen arm of the trial $(n=314)$. We first checked linearity of these two variables by fitting a Cox proportional hazard regression model with restricted cubic splines, and $\mathrm{H}: \mathrm{I}$ demonstrated significant nonlinearity. We used a polynomial function of $\mathrm{H}: \mathrm{I}$ to approximate the restricted cubic spline, and the final model was selected by comparing Cox regression models using Akaike Information Criterion. The resulting predictor from the final Cox regression model was then re-scaled into the range of $0-10$, which we refer to as the BCI. We further categorised BCI into three levels: low risk, $\mathrm{BCI}<5$; intermediate risk, $5 \leqslant \mathrm{BCI}<6.4$; high risk, $\mathrm{BCI} \geqslant 6.4$. These cut-offs were chosen such that the resulting proportions of low, intermediate and high risk groups were similar to those formed by the three categorical combination groups of $\mathrm{H}: \mathrm{I}$ and MGI. The endocrine-untreated arm in the Stockholm randomised trial was used as a testing cohort for BCI.

\section{Clinicopathological risk assessment}

The St Gallen's guidelines were used to assess the risk of recurrence in the ER-positive tamoxifen-treated and untreated patients, whereas Adjuvant! Online was used to assess 10-year risk of recurrence and survival for the ER-positive, node-negative patients using the following information: tumour grade, tumour size and age. Multivariate Cox proportional hazard regression models were used to assess the prognostic performance of Adjuvant! Online and BCI. 


\section{Statistical analysis}

The primary clinical endpoint used in data analysis was time to distant metastasis. Distant metastasis-free survival (DMFS) was defined as the time from diagnosis to first distant metastasis. Local/regional recurrences before distant metastasis were censored at the time of relapse. For analysis, data were censored at 15 years, as $>90 \%$ of the distant metastatic events occurred before this time point. Association of gene expression indices with the clinical endpoint was assessed by the Kaplan-Meier method with the use of log-rank test and Cox proportional hazard regression. The proportional hazard assumption was verified by scaled Schoenfeld residuals. Multivariate Cox proportional hazard regression models were used to assess whether gene expression indices provided prognostic information independent of traditional clinical and histopathological parameters. The hazard ratio for the continuous BCI score was calculated relative to a 5-unit increment except for in the multivariate analysis of $\mathrm{BCI}$ and Adjuvant! Online. To more accurately compare BCI with Adjuvant! Online, hazard ratios were calculated relative to an increment of their inter-quartile ranges (2.484 for BCI; 8 for Adjuvant! Online). All statistical procedures are conducted in the statistical software Statistica 9.1 (StatSoft Scandinavia AB, Uppsala, Sweden) and the free software environment R (version 2.11.1, http://www.r-project.org/).

\section{RESULTS}

\section{Patient and tumour characteristics}

The randomised Stockholm trial conducted during 1976 through 1990 examined the efficacy of adjuvant tamoxifen compared with no adjuvant treatment among postmenopausal women with earlystage breast cancer. From the 'low-risk' patient group (negative lymph nodes and tumour size $\leqslant 3 \mathrm{~cm}$ ) in this trial, a total of 808 FFPE tumour blocks were retrieved for molecular analysis. After pathological review, 37 cases had to be excluded because of insufficient tumour cells or only containing carcinoma in situ. Reportable gene expression data by real-time RT-PCR were obtained for all but two samples, leaving a total of 769 cases in the final analysis (Figure 1). This corresponds to a success rate of 99.7\% among the samples assayed. The ER-positive tamoxifentreated and untreated patients were examined in this study $(n=588)$. Further tumour characteristics of this cohort are summarised in Table 1.

\section{Association of the combined $\mathrm{H}: \mathrm{I}$ and MGI with distant metastasis and breast cancer-specific death}

The dichotomous $\mathrm{H}$ : I and MGI index was defined for each patient using pre-specified cut-off values ( $\mathrm{H}: \mathrm{I} 0.06$ and MGI 0 ) and a previously described combination algorithm (low risk, low MGI; intermediate risk, low $\mathrm{H}$ : I and high MGI; and high risk, high $\mathrm{H}: \mathrm{I}$ and high MGI) (Ma et al, 2008). Using this approach to estimate risk among the ER-positive tamoxifen-treated and untreated patients, the index was significantly associated with both time to first distant metastasis and breast cancer-specific death within these independent cohorts. In the tamoxifen-treated patients, more than $50 \%$ of the patients were classified as having a low risk of recurrence. Kaplan-Meier analysis demonstrated that tamoxifentreated patients assigned to the low-risk group had a rate of distant recurrence or death at 10 years of less than $3 \%$ (distant recurrence rate: $2.9 \%, 95 \%$ CI: $0.4-5.4$ (Figure $2 \mathrm{~A}$ ); rate of death: $2.3 \%, 95 \%$ CI: $0.1-4.5$ (data not shown)). The dichotomous H:I and MGI index also identified $23 \%$ of the tamoxifen-treated patients as intermediate risk and $18 \%$ as high risk. In the intermediate and high-risk groups, the Kaplan-Meier estimates of the rate of distant recurrence were $16.9 \%(95 \% \mathrm{CI}: 7.2-25.6)$ and $16.3 \%(95 \% \mathrm{CI}$ : 6.0-25.5; Figure 2A) and estimates of the 10-year rate of breast

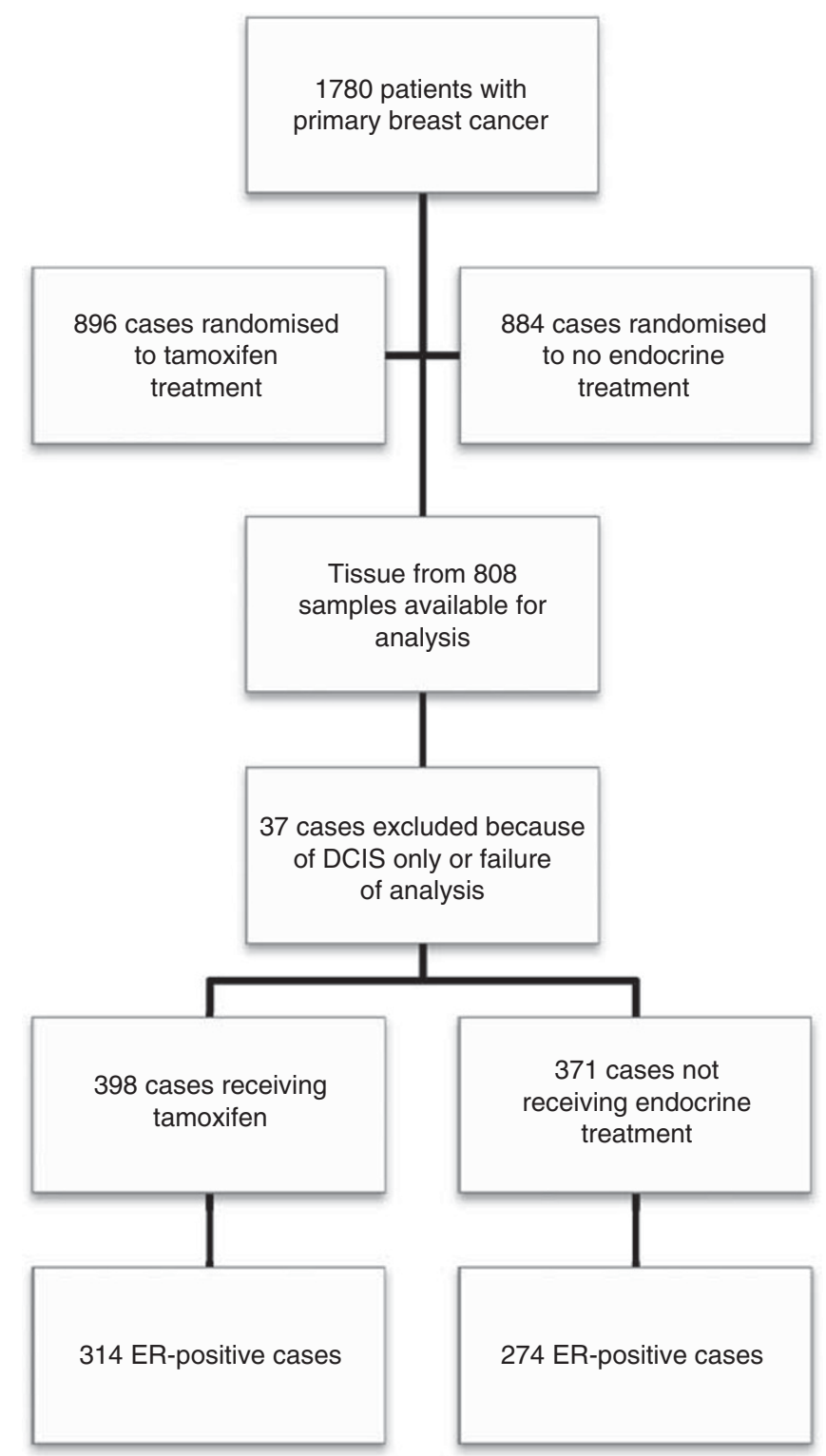

Figure I CONSORT diagram.

cancer-specific death were $13.8 \%$ (95\% CI: $5.0-21.9)$ and $11.0 \%$ (95\% CI: $2.3-18.9$ )

A multivariate Cox regression model including tumour grade, tumour size and the dichotomous H:I and MGI index confirmed that this risk classification was associated with distant recurrence independent of tumour size, grade, HER2 status and PR status in the tamoxifen-treated patients (Table 2). The dichotomous $\mathrm{H}: \mathrm{I}$ and MGI index was also prognostic of breast cancer-specific death independent of tumour size, grade, HER2 status and PR status (Supplementary Table 1).

The dichotomous $\mathrm{H}: \mathrm{I}$ and MGI index also demonstrated prognostic utility in the ER-positive untreated arm of the trial $(P=0.0004$; Figure 2B). Of the untreated cohort, $50 \%$ were classified as low risk, $27 \%$ as intermediate risk and $23 \%$ as high risk. The prediction of outcome for the untreated patients showed a similar pattern but with higher recurrence rates and death rates than in the tamoxifen-treated patients. In univariate Cox regression analysis, risk assessment with the dichotomous $\mathrm{H}$ : I and MGI index showed a statistical significance both for distant recurrence, as well as death due to the disease (data not shown). The rate of 


\section{$\underset{\text { OPEN }}{B} \mathbb{1}$}

breast cancer-specific death for the low-, intermediate- and high-risk groups in the untreated arm of the trial was 5.3\% (95\% CI: 1.4-9.0), 19.3\% (95\% CI: $9.3-28.3$ ) and 26.3\% (95\% CI: $14.4-36.7$ ).

In a multivariate analysis, the dichotomous $\mathrm{H}: \mathrm{I}+\mathrm{MGI}$ index was prognostic of distant recurrence (Table 3) and breast cancerspecific death (Supplementary Table 2) independent of tumour size, grade, HER2 status and PR status in the untreated cohort.

\section{Development and testing of $\mathrm{H}: \mathrm{I}+\mathrm{MGI}$ as a continuous index (BCI)}

To enable individual risk assessment of the risk of recurrence, we developed a continuous algorithm based on the dichotomous $\mathrm{H}$ : I and MGI index. Using the ER-positive tamoxifen-treated patients in this cohort as a training set, we developed a polynomial function

Table I Tumour characteristics for the 588 early-stage postmenopausal ER-positive breast cancer patients included in this study

\begin{tabular}{|c|c|c|c|c|}
\hline & \multicolumn{2}{|c|}{ Tamoxifen treated } & \multicolumn{2}{|c|}{ Untreated } \\
\hline & No. & $\%$ & No. & $\%$ \\
\hline \multicolumn{5}{|c|}{ Tumour size (mm) } \\
\hline$\leqslant 20$ & 256 & 82 & 223 & 81 \\
\hline$>20$ & 55 & 18 & 49 & 18 \\
\hline Unknown & 3 & I & 2 & । \\
\hline \multicolumn{5}{|l|}{ Tumour grade } \\
\hline I & 67 & 21 & 67 & 24 \\
\hline 2 & 209 & 67 & 172 & 63 \\
\hline 3 & 38 & 12 & 35 & 13 \\
\hline \multicolumn{5}{|l|}{ PR status } \\
\hline Negative & 109 & 35 & 107 & 39 \\
\hline Positive & 180 & 57 & 139 & 51 \\
\hline Unknown & 25 & 8 & 28 & 10 \\
\hline \multicolumn{5}{|l|}{ HER2 status } \\
\hline Negative & 272 & 87 & 238 & 87 \\
\hline Positive & 14 & 4 & 13 & 5 \\
\hline Unknown & 28 & 9 & 23 & 8 \\
\hline
\end{tabular}

Abbreviations: $\mathrm{HER} 2$ = human epidermal growth factor receptor $2 ; \mathrm{PR}=$ progesterone receptor.
Prognostic utility of the Breast Cancer Index

P-L Jerevall et al

Table 2 Multivariate Cox regression analysis of distant metastasis for tamoxifen-treated patients with oestrogen receptor-positive disease

\begin{tabular}{|c|c|c|c|}
\hline & Patients (n) & $\begin{array}{c}\text { Hazard ratio } \\
(95 \% \mathrm{CI})\end{array}$ & $P$-value \\
\hline \multicolumn{4}{|c|}{ Multivariate analysis without $\mathrm{H}: \mathrm{I}+\mathrm{MGl}$} \\
\hline \multicolumn{4}{|c|}{ Tumour grade } \\
\hline$N H G \mid$ & 54 & & \\
\hline $\mathrm{NHG} \|$ & 183 & $2.6(0.6-11.4)$ & 0.20 \\
\hline NHG III & 36 & $5.7(1.2-27.3)$ & 0.03 \\
\hline \multicolumn{4}{|l|}{ Tumour size } \\
\hline$\leqslant 20 \mathrm{~mm}$ & 225 & & \\
\hline$>20 \mathrm{~mm}$ & 48 & $1.0(0.4-2.5)$ & 0.98 \\
\hline \multicolumn{4}{|l|}{ HER2 } \\
\hline Negative & 259 & & \\
\hline Positive & 14 & $2.1(0.7-6.4)$ & 0.22 \\
\hline \multicolumn{4}{|l|}{$P R$} \\
\hline Negative & 104 & & \\
\hline Positive & 169 & $0.8(0.4-1.7)$ & 0.56 \\
\hline \multicolumn{4}{|c|}{ Multivariate analysis with $\mathrm{H}: 1+\mathrm{MGl}$} \\
\hline \multicolumn{4}{|c|}{ Tumour grade } \\
\hline $\mathrm{NHG} \mid$ & 54 & & \\
\hline$N H G \|$ & 183 & $1.5(0.3-7.0)$ & 0.59 \\
\hline NHG III & 36 & $2.0(0.4-10.7)$ & 0.44 \\
\hline \multicolumn{4}{|l|}{ Tumour size } \\
\hline$\leqslant 20 \mathrm{~mm}$ & 225 & & \\
\hline$>20 \mathrm{~mm}$ & 48 & $1.0(0.4-2.5)$ & 0.96 \\
\hline \multicolumn{4}{|l|}{ HER2 } \\
\hline Negative & 259 & & \\
\hline Positive & 14 & $1.6(0.5-5.3)$ & 0.42 \\
\hline \multicolumn{4}{|l|}{$P R$} \\
\hline Negative & 104 & & \\
\hline Positive & 169 & $0.8(0.4-1.8)$ & 0.62 \\
\hline $\mathrm{H}: 1+\mathrm{MGl}$ & & & 0.02 \\
\hline Low & 155 & & \\
\hline Intermediate & 67 & $4.2(1.5-12.1)$ & 0.007 \\
\hline High & 51 & $4.4(1.4-13.7)$ & 0.01 \\
\hline
\end{tabular}

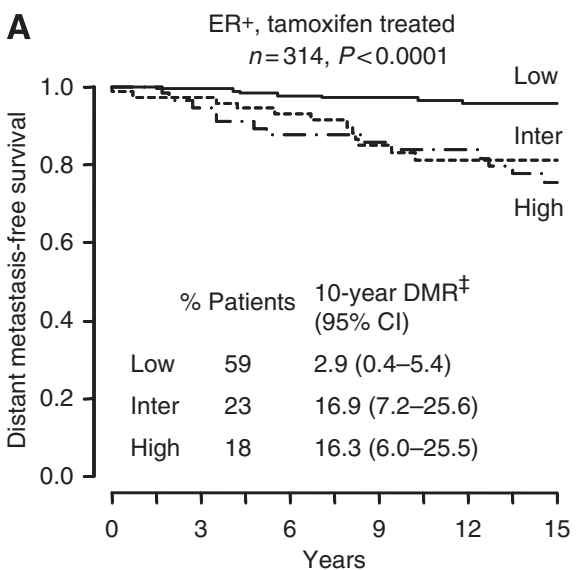

Figure 2 Kaplan-Meier estimates of likelihood of distant metastasis, stratified by the combined index of $\mathrm{H}$ : I and MGl. (A) ER-positive, tamoxifen-treated subcohort. (B) ER-positive, untreated subcohort. DMR $R^{\ddagger}$ denotes distant metastasis rate. 
Table 3 Multivariate Cox regression analysis of distant metastasis for untreated patients with oestrogen receptor positive disease

\begin{tabular}{|c|c|c|c|}
\hline & Patients (n) & $\begin{array}{c}\text { Hazard ratio } \\
(95 \% \mathrm{Cl})\end{array}$ & $P$-value \\
\hline \multicolumn{4}{|c|}{ Multivariate analysis without $\mathrm{H}: I+\mathrm{MGl}$ or $\mathrm{BCl}$} \\
\hline \multicolumn{4}{|c|}{ Tumour grade } \\
\hline NHG I & 53 & & \\
\hline NHG ॥ & 149 & $1.2(0.5-2.6)$ & 0.74 \\
\hline NHG III & 34 & $1.6(0.6-4.4)$ & 0.37 \\
\hline \multicolumn{4}{|l|}{ Tumour size } \\
\hline$\leqslant 20 \mathrm{~mm}$ & 190 & & \\
\hline$>20 \mathrm{~mm}$ & 46 & $3.0(1.6-5.6)$ & 0.0005 \\
\hline \multicolumn{4}{|l|}{ HER2 } \\
\hline Negative & 223 & & \\
\hline Positive & 13 & $3.0(1.1-8.3)$ & 0.03 \\
\hline \multicolumn{4}{|l|}{$P R$} \\
\hline Negative & 101 & & \\
\hline Positive & 135 & $1.3(0.7-2.4)$ & 0.41 \\
\hline \multicolumn{4}{|c|}{ Multivariate analysis with $\mathrm{H}: 1+M G l$} \\
\hline \multicolumn{4}{|c|}{ Tumour grade } \\
\hline NHG I & 53 & & \\
\hline NHG ॥ & 149 & $0.9(0.4-2.1)$ & 0.79 \\
\hline NHG III & 34 & $0.9(0.3-2.8)$ & 0.84 \\
\hline \multicolumn{4}{|l|}{ Tumour size } \\
\hline$\leqslant 20 \mathrm{~mm}$ & 190 & & \\
\hline$>20 \mathrm{~mm}$ & 46 & $2.8(1.5-5.3)$ & 0.001 \\
\hline \multicolumn{4}{|l|}{ HER2 } \\
\hline Negative & 223 & & \\
\hline Positive & 13 & $2.8(1.0-7.7)$ & 0.053 \\
\hline \multicolumn{4}{|l|}{$P R$} \\
\hline Negative & 101 & & \\
\hline Positive & 135 & $1.3(0.7-2.4)$ & 0.39 \\
\hline$H:|+M G|$ & & & 0.048 \\
\hline Low & 116 & & \\
\hline Intermediate & 62 & $1.8(0.8-3.9)$ & 0.17 \\
\hline High & 58 & $2.6(1.2-5.6)$ & 0.01 \\
\hline \multicolumn{4}{|c|}{ Multivariate analysis With $\mathrm{BCl}$ categories } \\
\hline \multicolumn{4}{|c|}{ Tumour grade } \\
\hline $\mathrm{NHG} I$ & 53 & & \\
\hline NHG $\|$ & 149 & $0.8(0.3-1.8)$ & 0.55 \\
\hline NHG III & 34 & $0.6(0.2-1.9)$ & 0.39 \\
\hline \multicolumn{4}{|l|}{ Tumour size } \\
\hline$\leqslant 20 \mathrm{~mm}$ & 190 & & \\
\hline$>20 \mathrm{~mm}$ & 46 & $3.0(1.5-5.6)$ & 0.001 \\
\hline \multicolumn{4}{|l|}{ HER2 } \\
\hline Negative & 223 & & \\
\hline Positive & 13 & $3.5(1.2-9.8)$ & 0.019 \\
\hline \multicolumn{4}{|l|}{$P R$} \\
\hline Negative & 101 & & \\
\hline Positive & 135 & $1.4(0.7-2.6)$ & 0.30 \\
\hline $\mathrm{BCl}$ & & & 0.001 \\
\hline Low & 122 & & \\
\hline Intermediate & 66 & $2.3(1.1-5.0)$ & 0.03 \\
\hline High & 48 & $4.7(2.1-10.8)$ & 0.0003 \\
\hline \multicolumn{4}{|c|}{ Multivariate analysis with continuous $B C l$ score } \\
\hline Tumour grade & & & 0.89 \\
\hline $\mathrm{NHG} I$ & 53 & & \\
\hline NHG ॥ & 149 & $1.3(0.4-3.8)$ & 0.69 \\
\hline NHG III & 34 & $1.1(0.3-4.4)$ & 0.91 \\
\hline
\end{tabular}

Table 3 (Continued)

\begin{tabular}{|c|c|c|c|}
\hline & Patients (n) & $\begin{array}{c}\text { Hazard ratio } \\
(95 \% \mathrm{Cl})\end{array}$ & $P$-value \\
\hline \multicolumn{4}{|l|}{ Tumour size } \\
\hline$\leqslant 20 \mathrm{~mm}$ & 190 & & \\
\hline$>20 \mathrm{~mm}$ & 46 & $2.1(1.1-4.2)$ & 0.03 \\
\hline \multicolumn{4}{|l|}{ HER2 } \\
\hline Negative & 223 & & \\
\hline Positive & 13 & $2.1(0.7-6.4)$ & 0.18 \\
\hline \multicolumn{4}{|l|}{$P R$} \\
\hline Negative & 101 & & \\
\hline Positive & 135 & $1.0(0.5-1.9)$ & 0.91 \\
\hline$B C l^{\mathrm{a}}$ & 236 & $7.5(2.4-23.6)$ & 0.0006 \\
\hline \multicolumn{4}{|c|}{ Multivariate analysis with $\mathrm{BCl}$ and adjuvant! online } \\
\hline Adjuvant! online $e^{b}$ & $246^{\prime}$ & $1.4(1.0-1.8)$ & 0.03 \\
\hline $\mathrm{BCl}^{\mathrm{b}}$ & 246 & $2.0(1.3-3.1)$ & 0.001 \\
\hline
\end{tabular}

Abbreviations: $\mathrm{BCl}=$ Breast Cancer Index; HER2 = human epidermal growth factor receptor 2; $\mathrm{MGI}=$ molecular grade index; $\mathrm{NHG}=$ Nottingham grade; $\mathrm{PR}=$ progesterone receptor ${ }^{\mathrm{a}} \mathrm{Analysis}$ of $\mathrm{BCl}$ as a continuous variable. The hazard ratio for $\mathrm{BCl}$ is calculated relative to a 5 -unit increment. ${ }^{\text {b}}$ To more accurately compare $\mathrm{BCl}$ to Adjuvant! Online, the calculated hazard ratios are relative to an increment of their inter-quartile ranges, 2.484 for $\mathrm{BCl}, 6$ for Adjuvant! Online.

to compute a continuous risk index from $\mathrm{H}$ : I and MGI, which we henceforth refer to as the BCI. Breast Cancer Index provides an individual risk score on the scale of $0-10$ for each patient, which has a continuous relationship with the rate of distant metastasis at 10 years (Figure 3A). For patient stratification using BCI, we also defined three risk groups using two cut-off points: $\mathrm{BCI}<5$, low risk; $\mathrm{BCI} \geqslant 5$ and $<6.4$, intermediate risk; $\mathrm{BCI} \geqslant 6.4$, high risk. $\mathrm{BCI}$ classified $59.6 \%$ of the tamoxifen-treated patients as having a low risk of recurrence (Kaplan-Meier estimates of the rate of distant recurrence: $1.7 \%, 95 \%$ CI: $0-3.5$; rate of death: $1.1 \%, 95 \%$ CI: 0-2.6 (Figure 3A; data not shown)). Breast Cancer Index also identified $22.0 \%$ of the tamoxifen-treated patients as intermediate risk and $18.4 \%$ as high risk. In the intermediate- and high-risk groups, the Kaplan-Meier estimates of the rate of distant recurrence were $17.8 \%$ (95\% CI: $7.6-26.8)$ and $20.0 \%(95 \%$ CI: 8.7-30.0) and estimates of the 10-year rate of breast cancerspecific death were $14.5 \%$ (95\% CI: $5.2-22.9)$ and $14.7 \%(95 \%$ CI: 4.7-23.6) (data not shown).

To test the performance of the BCI model, the ability of BCI to predict distant metastasis in the ER-positive patients in the untreated arm of the Stockholm trial was examined $(n=274)$. In these patients, 53,27 and $20 \%$ were classified as low, intermediate and high risk. The rate of distant metastasis at 10 years in these risk groups was $8.3 \%$ (95\% CI: $4.7-14.4$ ), $22.9 \%$ (95\% CI: $14.5-$ 35.2 ) and $28.5 \%$ (95\% CI: $17.9-43.6$ ), respectively (Figure $3 \mathrm{~B}$ ), and the rate of breast cancer-specific death was 5.1\% (95\% CI: $1.3-8.7$ ), $19.8 \%$ (95\% CI: $10.0-28.6$ ) and $28.8 \%$ (95\% CI: $15.3-40.2)$.

Breast Cancer Index was a strong prognostic factor for distant recurrence independent of tumour size, grade, PR status and HER2 status, although tumour size did contribute prognostic value (Table 3). Breast Cancer Index was also predictive of breast cancerspecific death applying a similar multivariate model (Supplementary Table 2).

\section{Clinicopathological risk assessment}

The risk of recurrence was also assessed in both ER-positive cohorts from the Stockholm trial using the St Gallen's guidelines. In the tamoxifen-treated cohort, $22 \%$ were classified as low risk 


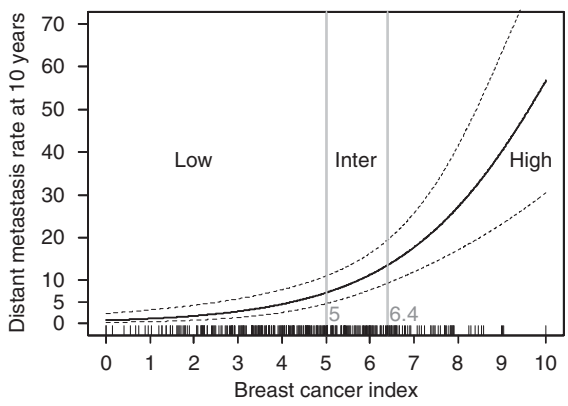

$$
\begin{array}{lcl} 
& \% \text { Patients } & 10 \text {-year DMR }{ }^{\ddagger}(95 \% \mathrm{Cl}) \\
\text { Low } & 60 & 1.7(0-3.5) \\
\text { Inter } & 22 & 17.8(7.6-26.8) \\
\text { High } & 18 & 20.0(8.7-30.0)
\end{array}
$$

B

ER+, untreated

$n=274, P<0.0001$

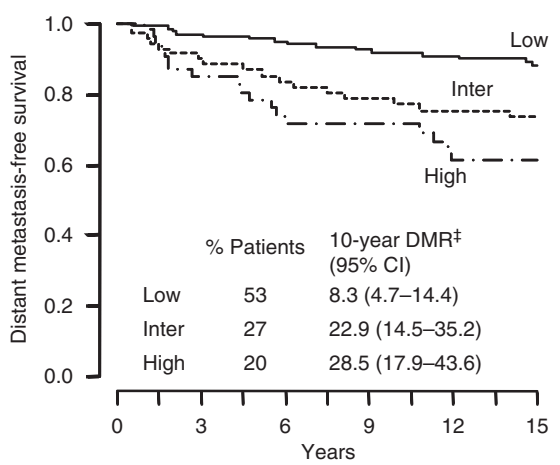

Figure 3 Breast Cancer Index predicts distant metastasis. A patient is in the low risk group, if $\mathrm{BCl}<5$, intermediate group if $5 \leqslant \mathrm{BCl}<6.4$, and high risk group if $\mathrm{BCl} \geqslant 6.4$. (A) Kaplan-Meier estimates of I 0 -year distant recurrence stratified by categorical $\mathrm{BCl}$ and distant metastasis rate at $\mathrm{I} 0$ years as a function of BCl based on ER-positive tamoxifen-treated patients from the Stockholm trial. (B) Kaplan-Meier estimates of likelihood of distant metastasis stratified by categorical $\mathrm{BCl}$ in the ER-positive untreated patients from the Stockholm trial. DMR ${ }^{\ddagger}$ denotes distant metastasis rate.

and $78 \%$ as intermediate risk with a rate of recurrence of $5.2 \%$ (95\% CI: -0.5 to 10.9$)$ and $8.5 \%$ (95\% CI: $4.9-12.1$ ), respectively. In the untreated cohort, $19 \%$ were classified as low risk and $81 \%$ as intermediate risk with a rate of recurrence of $8.8 \%(95 \% \mathrm{CI}: 1.4-$ 16.2 ) and $17.0 \%$ (95\% CI: $11.7-22.3)$ respectively.

The prognostic utility of BCI was also assessed in comparison with Adjuvant! Online, a web-based tool used to assess risk of recurrence and breast cancer-specific death based on clinicopathological information. In multivariate analyses, both BCI and Adjuvant! Online were significant predictors of distant recurrence and death (Table 3 and Supplementary Table 2).

\section{Correlation of BCI with traditional prognostic factors}

Comparisons between BCI and classic prognostic factors showed a correlation with tumour size and tumour grade, as well as HER2 status (Supplementary Table 3). Significantly more patients categorised into the low-risk group than in the high-risk group had tumours that were $\leqslant 20 \mathrm{~mm}$ in size, of a low grade and HER2 negative.

\section{DISCUSSION}

It was previously demonstrated that the combination of $\mathrm{H}: \mathrm{I}$ and MGI into a dichotomous index outperforms either index alone in predicting the risk of recurrence in ER-positive, node-negative breast cancer patients ( $M a$ et al, 2004, 2008). In this study, the prognostic performance of the dichotomous $\mathrm{H}$ : I and MGI index was validated in a large retrospective analysis of patients from the randomised Stockholm trial and a continuous risk model of $\mathrm{H}: \mathrm{I}$ and MGI (BCI) was developed and tested.

Consistent with previous reports, analysis in this cohort of 588 early stage, postmenopausal ER-positive breast cancer patients demonstrated that the combination of $\mathrm{H}$ : I and MGI was strongly associated with the risk of distant metastasis and death due to breast cancer (Ma et al, 2008). The combined H:I and MGI identified more than $50 \%$ of the patients to have a low 10 -year recurrence risk with fewer than $3 \%$ of the patients relapsing during this period of time. Additionally, the results from analysis of the ER-positive patients not treated with endocrine therapy suggested that the combined H:I and MGI also has prognostic utility in untreated patients. $\mathrm{H}: \mathrm{I}$ and MGI was shown to outperform tumour grade and PR status in a multivariate analysis, with tumour size still contributing significant prognostic value only in the untreated patient population. The results in this study thus confirmed the previous findings that the combined index performs well for prediction of breast cancer outcome, both in treated and untreated patients.

The tamoxifen-treated ER-positive, node-negative cohort from the Stockholm trial was used to develop the BCI algorithm. The BCI model was developed to assign a different index score for each patient, each associated with a different level of individualised risk of distant recurrence. The BCI scores were categorised into three levels of recurrence risk (low, intermediate and high) by using proportional values established with the dichotomous $\mathrm{H}: \mathrm{I}$ and MGI index. This entire cohort was used to train the algorithm in order to retain the entirety of the prognostic information available with this large, prospective trial and to maximise the accuracy of $\mathrm{BCI}$ to predict a distant metastatic recurrence. An initial test of the prognostic ability of BCI was carried out in the ER-positive patient cohort that was not treated with endocrine therapy and not used to develop BCI. In this untreated cohort, BCI provided similar prognostic utility compared with that of the combined $\mathrm{H}: \mathrm{I}$ and MGI in the untreated cohort. Specifically, BCI classified $53 \%$ of the patients into the low-risk group with a $8.3 \%$ risk of distant recurrence, whereas $\mathrm{H}: \mathrm{I}$ and MGI stratified $50 \%$ of the untreated, ER-positive patients into the low-risk group with a $9.5 \%$ risk of recurrence. In addition, these results also suggest that the prognostic utility of BCI extends into untreated patients.

In this study, BCI classified 53, 27 and $20 \%$ as low, intermediate and high risk. In contrast, traditional risk classification based on clinicopathological criteria, such as suggested by the St Gallen recommendations from 2005 (Goldhirsch et al, 2006), classifies a majority of ER-positive, node-negative breast cancer patients as being of intermediate risk, with few patients in the low- or highrisk groups. For the low-risk group with endocrine responsiveness, endocrine therapy remains the primary treatment, but for the corresponding intermediate group the treatment of choice is not completely clear, likely resulting in both over and undertreatment. With BCI, the intermediate risk group was reduced, and a significantly larger proportion of patients were predicted to have low risk of recurrence, suggesting additional chemotherapy to be unnecessary.

The prognostic performance of BCI was also compared with Adjuvant! Online. In a multivariate analysis, both BCI and Adjuvant! Online, which estimates risk based on clinicopathological information, retained predictive significance suggesting that they provide complementary information in the assessment of risk of recurrence and overall survival. 
There are at least two gene-expression profiling assays that were developed that identify breast cancer patients with a low risk of recurrence. The 70-gene profile MammaPrint (the Amsterdam signature; Agendia, BV; Amsterdam, Holland), which is based on a microarray platform, was developed as a predictor of 5-year risk of distant metastasis and stratifies patients into low or high risk. It was reported that MammaPrint classifies $\sim 40 \%$ of node-negative, ER-positive and -negative patients into the low-risk group with a 10 -year risk of recurrence of $13 \%$ in this cohort that was predominantly untreated $(<7 \%$ received chemotherapy and hormonal therapy) (van de Vijver et al, 2002). Oncotype DX (Genomic Health, Inc., Redwood City, CA, USA) is a quantitative RT-PCR assay of a panel of 21 genes, which uses a continuous recurrence score for risk assessment. For decision making in the clinical setting, three risk groups are defined, with risk assessment in the ER-positive, node-negative, tamoxifen-treated arm of the NSABP-B14 trial ranging from $7 \%$ in the low-risk to $31 \%$ in the high-risk group (Paik et al, 2004). Risk assessment in the validation cohort for the dichotomous $\mathrm{H}: \mathrm{I}$ and MGI index was consistent with these results, with $59 \%$ of the tamoxifen-treated and $50 \%$ of the untreated classified into the low-risk group with a 10 -year risk of recurrence of 3 and $10 \%$ respectively.

In this study we demonstrate that the combination of $\mathrm{H}: \mathrm{I}$ and MGI, either as dichotomous or as a continuous variable (i.e., BCI), is a significant prognostic for early breast cancer. Studies determining the prognostic and/or predictive properties of the individual components of BCI are also ongoing. For example, within the same cohort, HOXB13 protein expression was demonstrated to be associated with patient benefit for tamoxifen treatment (Jerevall et al, 2010). This suggests that in addition to the strong performance of BCI as a prognostic, its components may also have predictive properties. Further studies are warranted to determine whether these findings will extend to current standard of care of ER-positive patients receiving 5-10 years of aromatase inhibitors.

\section{CONCLUSIONS}

Taken together, this study validates the predictive performance of the dichotomous $\mathrm{H}: \mathrm{I}$ and MGI index in a retrospective analysis of postmenopausal early-stage breast cancer patients randomised to tamoxifen or no endocrine treatment. We have also developed and

\section{REFERENCES}

Carter CL, Allen C, Henson DE (1989) Relation of tumor size, lymph node status, and survival in 24740 breast cancer cases. Cancer 63: $181-187$

Desmedt C, Sotiriou C (2006) Proliferation: the most prominent predictor of clinical outcome in breast cancer. Cell Cycle 5: 2198-2202

Fan C, Oh DS, Wessels L, Weigelt B, Nuyten DS, Nobel AB, van't Veer LJ, Perou CM (2006) Concordance among gene-expression-based predictors for breast cancer. $N$ Engl J Med 355: 560-569

Galea MH, Blamey RW, Elston CE, Ellis IO (1992) The Nottingham Prognostic Index in primary breast cancer. Breast Cancer Res Treat 22: 207-219

Goetz MP, Suman VJ, Ingle JN, Nibbe AM, Visscher DW, Reynolds CA, Lingle WL, Erlander M, Ma XJ, Sgroi DC, Perez EA, Couch FJ (2006) A two-gene expression ratio of homeobox 13 and interleukin-17B receptor for prediction of recurrence and survival in women receiving adjuvant tamoxifen. Clin Cancer Res 12: 2080-2087

Goldhirsch A, Coates AS, Gelber RD, Glick JH, Thurlimann B, Senn HJ (2006) First-select the target: better choice of adjuvant treatments for breast cancer patients. Ann Oncol 17: 1772-1776

Goldhirsch A, Ingle JN, Gelber RD, Coates AS, Thurlimann B, Senn HJ (2009) Thresholds for therapies: highlights of the St Gallen International Expert Consensus on the primary therapy of early breast cancer 2009 Ann Oncol 20: 1319-1329

Goldstein LJ, Gray R, Badve S, Childs BH, Yoshizawa C, Rowley S, Shak S, Baehner FL, Ravdin PM, Davidson NE, Sledge Jr GW, Perez EA, Shulman tested a continuous risk index of $\mathrm{H}: \mathrm{I}$ and MGI, called BCI, for estimation of recurrence risk at the individual level. The results from this study suggest that BCI has significant prognostic utility in an untreated population. Breast Cancer Index has the ability to identify a large fraction $(>50 \%)$ of patients with a low risk of distant recurrence at 10 years more accurately than using traditional risk assessment. These results suggest that BCI may help clinicians to make better informed treatment decisions and spare toxic chemotherapy for a large group of breast cancer patients.

\section{ACKNOWLEDGEMENTS}

This study was supported by grants from the Swedish Cancer Society, the Swedish Research Council (K2008-54X-20639-01-3) (to OS), King Gustaf V Jubilee Fund, National Cancer Institute (RO1-1CA112021) (to DCS), the Avon Foundation (to DCS), and bioTheranostics. We thank Yen Tran and Thao Ho for excellent technical assistance.

\section{Conflict of interest}

HL, RS, NCK and MGE are employees of and stockholders in bioTheranostics Inc., X-JM was an employee of bioTheranostics, Inc. X-JM, MGE and DCS are named inventors on a patent for the technology described herein.

\section{Author contributions}

X-JM and MGE conceived of the study. P-LJ, X-JM, MGE and OS participated in the study design and coordination. LS, TF and BN provided the study material and clinical information, and collected laboratory data on the study patients. P-LJ, RS, DCS and BH performed the laboratory work. P-LJ, X-JM, HL, MGE and OS performed the statistical analyses. P-LJ, X-JM, HL and NCK participated in interpreting results and drafting the manuscript. MGE and OS provided critical revision of the manuscript. All authors read and approved the final manuscript.

Supplementary Information accompanies the paper on British Journal of Cancer website (http://www.nature.com/bjc)
LN, Martino S, Sparano JA (2008) Prognostic utility of the 21-gene assay in hormone receptor-positive operable breast cancer compared with classical clinicopathologic features. J Clin Oncol 26: 4063-4071

Jerevall PL, Brommesson S, Strand C, Gruvberger-Saal S, Malmstrom P, Nordenskjold B, Wingren S, Soderkvist P, Ferno M, Stal O (2008) Exploring the two-gene ratio in breast cancer - independent roles for HOXB13 and IL17BR in prediction of clinical outcome. Breast Cancer Res Treat 107: $225-234$

Jerevall PL, Jansson A, Fornander T, Skoog L, Nordenskjold B, Stal O (2010) Predictive relevance of HOXB13 protein expression for tamoxifen benefit in breast cancer. Breast Cancer Res 12: R53

Ma XJ, Hilsenbeck SG, Wang W, Ding L, Sgroi DC, Bender RA, Osborne CK, Allred DC, Erlander MG (2006) The HOXB13:IL17BR expression index is a prognostic factor in early-stage breast cancer. J Clin Oncol 24: $4611-4619$

Ma XJ, Salunga R, Dahiya S, Wang W, Carney E, Durbecq V, Harris A, Goss P, Sotiriou C, Erlander M, Sgroi D (2008) A five-gene molecular grade index and HOXB13:IL17BR are complementary prognostic factors in early stage breast cancer. Clin Cancer Res 14: 2601-2608

Ma XJ, Wang Z, Ryan PD, Isakoff SJ, Barmettler A, Fuller A, Muir B, Mohapatra G, Salunga R, Tuggle JT, Tran Y, Tran D, Tassin A, Amon P, Wang W, Wang W, Enright E, Stecker K, Estepa-Sabal E, Smith B, Younger J, Balis U, Michaelson J, Bhan A, Habin K, Baer TM, Brugge J, 
$\underset{\text { OPEN }}{\mathrm{BJC}} \cap$

Haber DA, Erlander MG, Sgroi DC (2004) A two-gene expression ratio predicts clinical outcome in breast cancer patients treated with tamoxifen. Cancer Cell 5: 607-616

Paik S, Shak S, Tang G, Kim C, Baker J, Cronin M, Baehner FL, Walker MG, Watson D, Park T, Hiller W, Fisher ER, Wickerham DL, Bryant J, Wolmark N (2004) A multigene assay to predict recurrence of tamoxifen-treated, node-negative breast cancer. N Engl J Med 351: $2817-2826$

Press MF, Bernstein L, Thomas PA, Meisner LF, Zhou JY, Ma Y, Hung G, Robinson RA, Harris C, El-Naggar A, Slamon DJ, Phillips RN, Ross JS, Wolman SR, Flom KJ (1997) HER-2/neu gene amplification characterized by fluorescence in situ hybridization: poor prognosis in node-negative breast carcinomas. J Clin Oncol 15: 2894-2904

Ravdin PM, Siminoff LA, Davis GJ, Mercer MB, Hewlett J, Gerson N, Parker HL (2001) Computer program to assist in making decisions about adjuvant therapy for women with early breast cancer. J Clin Oncol 19: $980-991$

Rutqvist LE, Johansson H (2007) Long-term follow-up of the randomized Stockholm trial on adjuvant tamoxifen among postmenopausal patients with early stage breast cancer. Acta Oncologica (Stockholm, Sweden) 46: $133-145$

van't Veer LJ, Bernards R (2008) Enabling personalized cancer medicine through analysis of gene-expression patterns. Nature 452: 564-570

van de Vijver MJ, He YD, van't Veer LJ, Dai H, Hart AA, Voskuil DW, Schreiber GJ, Peterse JL, Roberts C, Marton MJ, Parrish M, Atsma D, Witteveen A, Glas A, Delahaye L, van der Velde T, Bartelink H, Rodenhuis S, Rutgers ET, Friend SH, Bernards R (2002) A gene-expression signature as a predictor of survival in breast cancer. $N$ Engl J Med 347: $1999-2009$

Wrange O, Nordenskjöld B, Gustafsson JA (1978) Cytosol estradiol receptor in human mammary carcinoma: an assay based on isoelectric focusing in polyacrylamide gel. Anal Biochem 85: 461-475

cc)(1) (2) This work is licensed under the Creative Commons cc. $\mathrm{BY}$ NG SA Attribution-NonCommercial-Share Alike 3.0 Unported License. To view a copy of this license, visit http:// creativecommons.org/licenses/by-nc-sa/3.0/ 